\title{
Extrinsic photoconduction and Rydberg states due to a methyl iodide impurity in xenon
}

\author{
I. T. Steinberger and U. Asaf \\ Racah Institute of Physics, The Hebrew University, Jerusalem 91904, Israel \\ G. Ascarelli \\ Department of Physics, Purdue University, West Lafayette, Indiana 47907
}

R. Reininger

Hamburger Synchrotronstrahlungslabor at Deutsches Elektronen-Synchrotron DESY, Notkestrasse 85, 2000

Hamburg 52, Federal Republic of Germany

G. Reisfeld and M. Reshotko

Racah Institute of Physics, The Hebrew University, Jerusalem 91904, Israel

(Received 12 April 1990; revised manuscript received 1 May 1990)

\begin{abstract}
Photoconductivity in $\mathrm{Xe}$ due to a $\mathrm{CH}_{3} \mathrm{I}$ impurity is presented as a function of incident photon energy at $\mathrm{Xe}$ number densities with the range $(3-15) \times 10^{19} \mathrm{~cm}^{-3}$, with constant $\mathrm{CH}_{3} \mathrm{I}$ concentrations. The Xe absorption spectrum between 9.5 and $10.6 \mathrm{eV}$, including both the strongly perturbed allowed transitions and molecular bands associated with forbidden lines, appears as dips in the photocurrent. At photon energies below $9.5 \mathrm{eV}$ the photocurrent increases exponentially with photon energy, and members of the $n d$ and $n s$ Rydberg series of $\mathrm{CH}_{3} \mathrm{I}$ appear as steps. From the shift of the step positions with density the electron scattering length in xenon was determined as $a=-0.324 \mathrm{~nm}$. The photocurrent versus photon energy curves shift more strongly with density than the superimposed Rydberg lines as a consequence of adiabatic polarization effects. The results provide clear evidence that photoconduction below the vertical ionization potential takes place by ionization of Rydberg states.
\end{abstract}

Molecular Rydberg states perturbed by an ambient rare gas ( $\mathrm{He}, \mathrm{Ne}, \mathrm{Ar}, \mathrm{Kr}$ ) (Refs. 1-4) or hydrogen ${ }^{5}$ were studied recently with perturber densities up to about $10^{21}$ $\mathrm{cm}^{-3}$. The higher Rydberg levels of the molecular solute $\left(\mathrm{CH}_{3} \mathrm{I}\right.$ or $\left.\mathrm{C}_{6} \mathrm{H}_{6}\right)$ were observed to broaden and shift with the increase of perturber density. The energy shift was analyzed on the basis of the Fermi theory, ${ }^{6}$ with a modification by Alekseev and Sobel'man ${ }^{7}$ as to the effect of polarization $\Delta_{\mathrm{pol}}$ by the positive core. The basic equation is

$$
\Delta=\Delta_{\mathrm{sc}}+\Delta_{\mathrm{pol}}
$$

where $\Delta$ is the asymptotic shift of the Rydberg states and $\Delta_{\text {sc }}$ the shift due to the interaction of the optical electron with the neutral atoms of the ambient gas.

For rare gases as perturbers, ${ }^{1-4}$ absorption spectroscopy was supplemented with the recording of photoconductivity excitation spectra. ${ }^{3}$ The Rydberg series limit determined from the absorption spectra is equal to the vertical ionization potential $I_{v}$, of the molecular solute in the perturber medium, while the observed photoionization thresholds were identified with an adiabatic ionization potential $I_{\mathrm{ad}}$. For $\mathrm{CH}_{3} \mathrm{I}$ and $\mathrm{C}_{6} \mathrm{H}_{6}$ the perturbers $\mathrm{He}, \mathrm{Ne}$, and $\mathrm{H}_{2}$ caused a blueshift of $I_{v}$, i.e., $d I_{v} / d \rho>0, \rho$ being the number density. For the same solutes in $\mathrm{Ar}$ and $\mathrm{Kr}$ the authors of Refs. $1-4$ found $d I_{v} / d \rho<0$. On the other hand, for all cases, $d I_{\mathrm{ad}} / d \rho<0$ and $\left|d I_{\mathrm{ad}} / d \rho\right|>\left|d I_{v} / d \rho\right|$. Köhler et al. ${ }^{3}$ described this observation by writing

$$
\Delta I_{\mathrm{ad}}=\Delta_{\mathrm{sc}}+P^{+}
$$

where $\boldsymbol{P}^{+}$represents the "adiabatic polarization energy." The difference between $P^{+}$and $\Delta_{\text {pol }}$ is related to the different time scales involved: During the absorption process $\left(\approx 10^{-16} \mathrm{sec}\right)$ the polarization is purely electronic. However, photoconduction below the vertical ionization limit is a result of ionization of a Rydberg state whose lifetime is of the order of $10^{-12} \mathrm{sec}$, thus ${ }^{3}$ some displacement of polarized perturber atoms towards the ionized solute molecule can also take place, yielding a considerable contribution to $P^{+}$. Therefore, $P^{+}<\Delta_{\text {pol }}<0$. On the other hand, $\Delta_{\text {sc }}$ in Eqs. (1) and (2) may be either positive $\left(\mathrm{He}, \mathrm{Ne}, \mathrm{H}_{2}\right)$ or negative $(\mathrm{Ar}, \mathrm{Kr}$ ), depending on the sign of the electron scattering length [see Eq. (5) below].

No molecular Rydberg shift studies have been hitherto reported using xenon as the perturber, since its strong absorption lines, above $8.436 \mathrm{eV}$, overlap and mask large parts of Rydberg spectra of many molecules.

In this work photocurrents were measured between two parallel gold electrodes (distance: $1 \mathrm{~mm}$ ) sputtered onto the inner surface of a $\mathrm{MgF}_{2}$ disk, serving as front window of the experimental cell. Indium plated stainless-steel screw heads were pressed onto the electrodes by springs. The cell was made of stainless steel with standard miniconflat flanges and copper gaskets; it could be evacuated down to about $10^{-6} \mathrm{~Pa}$ and withstand pressures from the inside up to $10^{7} \mathrm{~Pa}$. Synchrotron radiation at Hamburger Synchrotronstrahlungslabor (HASYLAB) monochromated by means of the HONORMI experimental station, ${ }^{8}$ entered the cell in the gap between the two surface electrodes. All photoconduction excitation spectra presented in this communication were taken at room temperature, 
with a resolution of $0.1 \mathrm{~nm}$ : $300 \mathrm{~V}$ dc were applied between the electrodes.

The experiments to be described are part of a more extensive study of the $\mathrm{CH}_{3} \mathrm{I} / \mathrm{Xe}$ system and pertain to host densities $\rho<2.5 \times 10^{20} \mathrm{~cm}^{-3}$. In order to have pronounced impurity photocurrents, high $\mathrm{CH}_{3}$ I concentration was essential. We will present results for the concentration of $c=1.1 \%$; for $c=0.06 \%$ the results were very similar though the currents were smaller and the steps (see below) somewhat less well resolved.

Figure 1 shows photoconductivity excitation spectra at various number densities. A broad envelope can be seen with a prominent pattern of dips superimposed. The onset of the photocurrent (i.e., the first rise of the envelope) shifts to lower energies with increasing density but the dip positions do not change appreciably. The known xenon atomic lines, ${ }^{9}$ including forbidden ones, are marked on the figure and so are the positions of the main maxima, as observed in absorption spectra by Laporte, Subtil, and Castex ${ }^{10}$ at a density of $2.58 \times 10^{20} \mathrm{~cm}^{-3}$ in a special absorption cell of $8.2-\mu \mathrm{m}$ length.

The interpretation of these features is straightforward. The envelope is due to extrinsic photoconduction, i.e., caused by electrons freed from the solute molecules by the photons [the photoconduction onset of $\mathrm{Xe}$ is, for the density range presented, at about $11 \mathrm{eV}$ (Ref. 12)]. However, absorption by the xenon host efficiently competes with the solute for the incident photons, causing dips at the positions of the absorption lines and bands.

It should be noted that the $n=7,8$, and 9 members of the $n d^{\prime}$ series of $\mathrm{CH}_{3} \mathrm{I}$ gas are at $9.58,9.76$, and $9.87 \mathrm{eV}$, respectively ${ }^{11}$ and peaks can be seen in Fig. 1 near these positions. However, such an assignment is rather tenta-

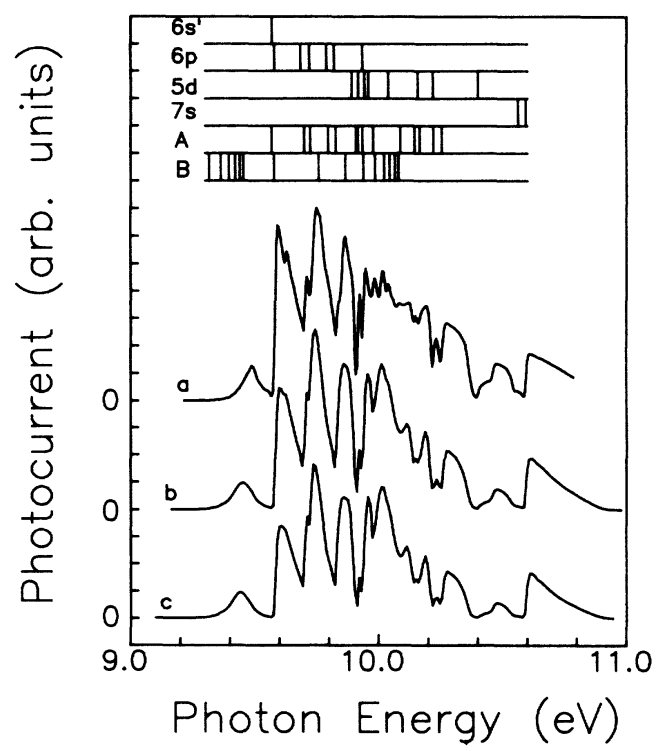

FIG. 1. Photocurrent as a function of photon energy in xenon gas with $1.1 \%$ methyl iodide impurity. Number densities in units of $10^{19} \mathrm{~cm}^{-3}: a, 4.68 ; b, 11.8 ; c, 14.7$. In the inset $6 s^{\prime}, 6 p$, $5 d$, and $7 s$ are atomic lines of xenon from Ref. 9, $A$ shows perturbed xenon lines from Ref. 10 , and $B$ shows $n d$ and $n d^{\prime}$ Rydberg lines of methyl iodide from Ref. 11 . tive because the spectrum can also be interpreted on the basis of the xenon lines only. Identification of higher members of the $n d^{\prime}$ series is even more dubious.

The low-energy region $(h v<9.5 \mathrm{eV})$ of Fig. 1 is shown in Fig. 2 on a semilogarithmic plot, without vertical staggering. It is seen that the photocurrent increases with density. In effect, this increase (at fixed photon energy) is linear with $\mathrm{Xe}$ density. The currents are only very slightly dependent on the $\mathrm{CH}_{3} \mathrm{I}$ density at the concentration considered, because of practically total absorption.

The semilogarithmic plots of Fig. 2 imply that, apart from the step pattern, the measured current $I$ satisfies, for $E<E_{0}$, Eq. (3):

$$
I=I_{0} \exp \left(\frac{E-E_{0}}{w}\right)
$$

Here $E$ is the photon energy, $E_{0}$ a characteristic energy (not determined from the present experiments), and $w$ is the inverse slope of the semilogarithmic plots. $w$ was found to increase from $0.029 \mathrm{eV}$ for $\rho=3.06 \times 10^{19} \mathrm{~cm}^{-3}$ to $0.033 \mathrm{eV}$ at $\rho=14.7 \times 10^{19} \mathrm{~cm}^{-3}$. The reason for this behavior is not yet understood; in all cases $w>k_{B} T, k_{B}$ being the Boltzmann constant and $T$ the absolute temperature. At higher densities (not shown) the step structure disappears (because of strong broadening) but the rise of the photocurrent stays exponential, with $w$ values increasing to about $0.06 \mathrm{eV}$ at $\rho=4 \times 10^{20} \mathrm{~cm}^{-3}$.

Comparison with absorption spectra ${ }^{11}$ of pure $\mathrm{CH}_{3} \mathrm{I}$ shows that the steps have to be attributed to nd and $n s$ Rydberg states of the methyl iodide molecule (Fig. 2). Since the steps could not be further resolved, we defined their positions as the minima of the first derivative $d I / d E$ of the spectra (not presented). Figure 3 shows the density

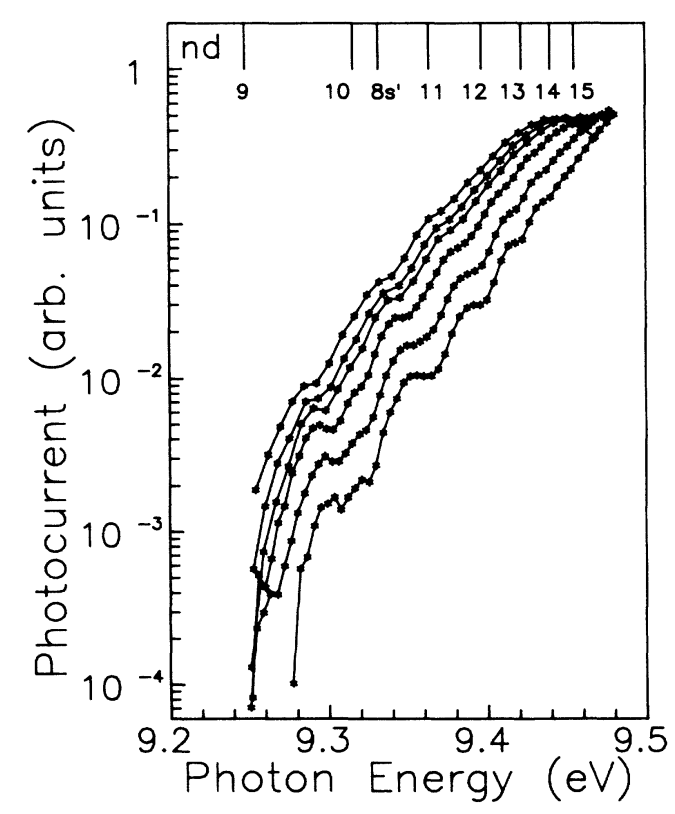

FIG. 2. Semilogarithmic plot of photocurrent vs photon energy spectra of xenon gas with $1.1 \%$ methyl iodide impurity. Number densities in units of $10^{19} \mathrm{~cm}^{-3}$, from bottom to top: $3.06,4.68,7.75,9.97,11.8$, and 14.7 . 


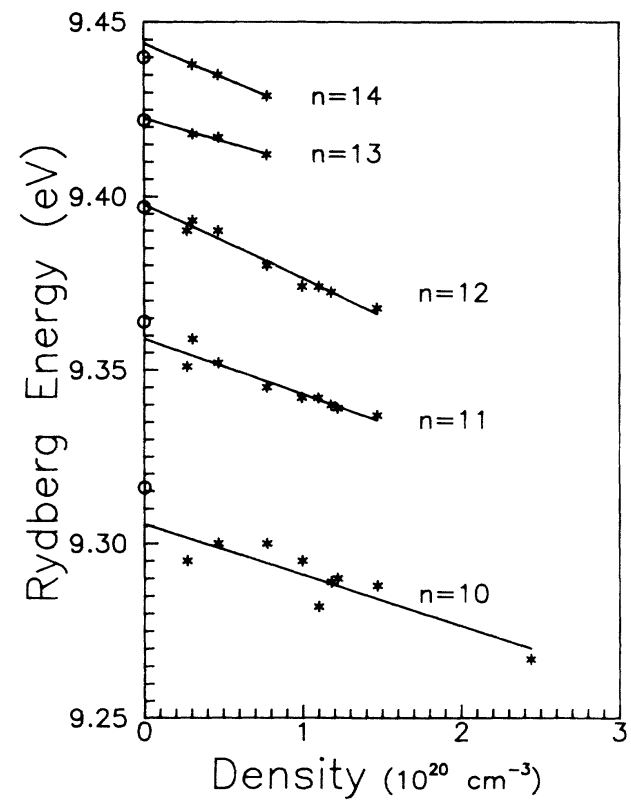

FIG. 3. Density dependence of the Rydberg line positions observed as steps in Fig. 2. See text. The zero-density values are taken from Ref. 11 and are not used in the linear regression fits. The differences in the slopes are due to the experimental error.

dependence the positions of five Rydberg lines as a function of the number density $\rho$.

We note that this is the first observation of high $n \mathrm{CH}_{3} \mathrm{I}$ Rydberg levels perturbed by xenon. Their study by absorption would be extremely difficult, since absorption by xenon is still strong in this region. In works on $\mathrm{CH}_{3} \mathrm{I}$ in other rare gases, it was inferred ${ }^{3}$ that below the vertical ionization potential $I_{r}$, photoconduction takes place by ionization of Rydberg levels, but no steps on the photoconductivity excitation spectrum, indicating the existence of the Rydberg levels, were seen. Similar spectra of aromatic molecule impurities in various liquid hydrocarbons ${ }^{13,14}$ did exhibit steps, but the identification of the respective Rydberg states was not clear. By way of contrast, in the present work steps were seen at positions very near to Rydberg levels of the free solute molecule, and the steps shift regularly with increasing density. These facts demonstrate clearly that photoconduction is due to ionization of Rydberg states.

The data were analyzed by means of Eqs. (1) and (2), as in previous works ${ }^{1-5}$ (Table I). The shift rate of $I_{r}$, $d I_{i} / d \rho \equiv \Delta / \rho$, was determined by taking the average shift rate of the Rydberg states with $n=10-14$, i.e., the average of the slopes of Fig. 3. The polarization contribution [Eq. (1)] of the positive core was calculated from the expression ${ }^{4,7}$

$$
\Delta_{\mathrm{pol}}=-10.78\left(\alpha e^{2} / 2\right)^{2 / 3}(\hbar v)^{1 / 3} \rho,
$$

TABLE I. Total shift rate $\Delta / \rho$, polarization shift rate $\Delta_{\mathrm{po}} / \rho$, scattering shift rate $\Delta_{\mathrm{sc}} / \rho$, adiabatic ionization shift rate $d I_{\mathrm{ad}} / d \rho$, and adiabatic polarization shift rate $P^{+} / \rho$, all in units of $10^{-23}$ $\mathrm{eV} \mathrm{cm}{ }^{3}$. Scattering length $a$ in $\mathrm{nm}$.

\begin{tabular}{llllll}
\hline \hline$\Delta / \rho$ & $\Delta_{\mathrm{poo}} / \rho$ & $\Delta_{\mathrm{sc}} / \rho$ & $a$ & $\frac{d I_{\mathrm{ad}}}{d \rho}$ & $P^{+} / \rho$ \\
\hline-16.8 & -1.29 & -15.5 & -0.324 & -55.2 & -40 \\
$-16.1^{\mathrm{a}}$ & & $-14.8^{\mathrm{b}}$ & $-0.310^{\mathrm{b}}$ & & $-59^{\mathrm{c}}$ \\
$-16.7^{\mathrm{d}}$ & & $-15.4^{\mathrm{e}}$ & $-0.323^{\mathrm{e}}$ & & \\
\hline \hline
\end{tabular}

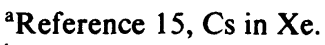

${ }^{b}$ Recalculated from Ref. 15 using $\Delta_{\mathrm{pol}} / \rho=1.29 \times 10^{-23} \mathrm{eV} \mathrm{cm}^{3}$.

'Calculated according to Ref. 3, using parameters for Xe-Xe potentials from Refs. 17-19.

${ }^{\mathrm{d}}$ Reference $16, \mathrm{Rb}$ in $\mathrm{Xe}$.

${ }^{\mathrm{e}}$ Calculated from Ref. 16 using $\Delta_{\mathrm{pol}} / \rho=-1.29 \times 10^{-23} \mathrm{eV} \mathrm{cm}$.

where $\alpha$ is the atomic polarizability of xenon $(\alpha=4.04$ $\left.\times 10^{-24} \mathrm{~cm}^{3}\right),{ }^{20} e$ the electronic charge, $\hbar$ the reduced Planck constant, $v$ the relative thermal velocity, and $\rho$ the number density. We determined $d I_{\mathrm{ad}} / d \rho$ by noting the photoconductivity threshold at each density. ${ }^{3}$ The scattering length $a$ was found from the formula ${ }^{6}$

$$
\Delta_{\mathrm{sc}}=\left(2 \pi \hbar^{2} / m\right) a \rho,
$$

with $m$ representing the electron mass. Results obtained from Rydberg shifts of alkali atom-doped xenon are also presented for comparison; they are reasonably consistent with our data, and so are results on other rare gases with $\mathrm{CH}_{3}$ I solute. ${ }^{3}$

We note that calculating the value of $I_{\text {ad }}$ from $d I_{\text {ad }} / d \rho$ (Table I) for zero density (i.e., pure $\mathrm{CH}_{3} \mathrm{I}$ ) gives the value $9.31 \mathrm{eV}$. For the other rare gases data indicate ${ }^{21}$ that $I_{\text {ad }}$ extrapolates at zero density to $9.48 \mathrm{eV}$. The difference seems to be due to the much smaller impurity concentrations employed in previous works. ${ }^{1-4}$

The observed dependence of the photocurrent (at fixed photon energies) on the density, taking into account the electron mobility values, ${ }^{22}$ can be shown to be in accord with a bimolecular ionization process involving a $\mathrm{CH}_{3} \mathrm{I}$ Rydberg state and a Xe atom.

This work confirms interpretations of other absorption and photoconductivity spectra, ${ }^{1-4}$ with several additional features, namely the observed exponential increase of the photocurrent with photon energy, the density dependence of the slope of the semilogarithmic plots, and the linear density dependence of the photocurrent at fixed photon energies. While several models may be considered, these must be tested by means of additional measurements.

This work was supported by Grant No. 85-0080 from the United States-Israel Binational Science Foundation, Jerusalem, Israel.
${ }^{1}$ A. M. Köhler, R. Reininger, V. Saile, and G. L. Findley, Phys. Rev. A 33, 771 (1986).

${ }^{2}$ A. M. Köhler, R. Reininger, V. Saile, and G. L. Findley, Phys. Rev. A 35, 79 (1987).
${ }^{3}$ A. M. Köhler, V. Saile, R. Reininger, and G. L. Findley, Phys. Rev. Lett. 60, 2727 (1988).

${ }^{4}$ R. Reininger, E. Morikawa, and V. Saile, Chem. Phys. Lett. 159, 276 (1989). 
${ }^{5}$ U. Asaf, W. S. Felps, K. Rupnik, S. P. McGlynn, and G. Ascarelli, J. Chem. Phys. 91, 5170 (1989).

${ }^{6}$ E. Fermi, Nuovo Cimento 11, 157 (1934).

${ }^{7}$ V. A. Alekseev and I. I. Sobel'man, Zh. Eksp. Teor. Fiz. 49, 1274 (1965) [Sov. Phys. JETP 22, 882 (1966)].

${ }^{8}$ V. Saile, P. Gürtler, E. E. Koch, A. Kozevnikov, M. Skibowski, and W. Steinmann, Appl. Opt. 15, 2559 (1976).

${ }^{9}$ C. E. Moore, Atomic Energy Levels, U.S. National Bureau of Standards Circulation No. 467 (U.S. GPO Washington, D.C., 1983), Vol. 3, p. 113.

${ }^{10}$ P. Laporte, J.-L. Subtil, and M.-C. Castex, in Spectral Line Shapes 2 (Walter and Gruyter \& Co., Berlin, 1983).

${ }^{11}$ H. T. Wang, W. S. Felps, G. L. Findley, A. R. P. Rau, and S. P. McGlynn, J. Chem. Phys. 67, 3940 (1977).

${ }^{12}$ R. Reininger, U. Asaf, I. T. Steinberger, V. Saile, and P. Laporte, Phys. Rev. B 28, 3193 (1983).

${ }^{13}$ R. A. Holroyd, J. M. Preses, E. H. Böttcher, and W. F. Schmidt, J. Phys. Chem. 88, 744 (1984).

${ }^{14}$ K. Nakagawa, A. Ejiri, M. Nishikawa, and K. Kimura, Chem.
Phys. Lett. 155, 278 (1989).

${ }^{15}$ D. K. L. Tan and S. Y. Ch'en, Phys. Rev. A 2, 1124 (1970).

${ }^{16}$ K. H. Weber and K. Niemax, Z. Phys. A 307, 13 (1982).

${ }^{17}$ P. M. Dehmer and S. T. Pratt, in Photophysics and Photochemistry in the Vacuum Ultraviolet, edited by S. P. McGlynn, G. L. Findley, and R. H. Huebner, NATO Advanced Study Institute Series C Vol. 142 (Reidel, Dodrecht, 1984).

${ }^{18}$ J. A. Barker, R. O. Watts, J. K. Lee, T. P. Schafer, and Y. T. Lee, J. Chem. Phys. 61, 3081 (1974).

${ }^{19}$ P. M. Dehmer and J. L. Dehmer, J. Chem. Phys. 68, 3462 (1978).

${ }^{20}$ A. Dalgarno and A. E. Kingston, Proc. R. Soc. London Ser. A 259, 424 (1961).

${ }^{21}$ A. M. Köhler, Ph.D. thesis, Hamburg University, 1987 (unpublished).

${ }^{22}$ S. S. S. Huang and G. R. Freeman, J. Chem. Phys. 68, 1355 (1978). 